\title{
Short turning pattern for relieving metro congestion during peak hours: the substance coherence of Shanghai, China
}

\author{
Xueqing Ding ${ }^{1,2}$, Shituo Guan ${ }^{3}$, Daniel Jian Sun ${ }^{1,2,4^{*}}$ (D) and Limin Jia ${ }^{4}$
}

\begin{abstract}
Introduction: Urban metro system generally has to deal with intractable heavily passenger loading during peak hours, in which demands are extreme huge in certain stations. However, overcrowding doesn't ubiquitously exist for all stations and mitigation measures have to be carried out on purpose, respectively. Because of the restrictions on operational costs and avoiding transportation resources wasting, simply increasing dispatch frequency is not rational to solve the problem.

Methods: Short turning pattern has been proved to be an efficient way to solve the issue, which had been mainly used in urban ground public transport systems. This paper applied short turning pattern to urban metro system and relaxed constraints of the turning-back facility. A mathematical model is proposed to determine the short turning parameters, during which a load factor was introduced as a measurement of overcrowding condition. An empirical case from Shanghai Metro Line 2 was incorporated to demonstrate the effectiveness of the proposed model.
\end{abstract}

Results: The results indicated that the short turning route from Beixinjing to Longyang Rd. in Shanghai Metro Line 2 could effectively relieve overcrowding within the heavy traffic demand zones.

Conclusions: Findings of this study could provide valuable suggestions in metro system administration for potential improvement on the operational performance during peak hours.

Keywords: Short turning, Relieve congestion, Metro system, Peak hours

\section{Introduction}

Urban metro system is a high-capacity public transport mode aiming at providing convenient and efficient services for passengers $[1,2]$, which plays an essential role in commuting traffic, especially in megacities, such as New York, London, Tokyo, and Shanghai and so on. In China, metro systems have been built in many cities during recent years, for example, Shanghai has more than $600 \mathrm{~km}$ mileage of subway and light rails (by 2016), which is still not able to satisfy the growing demand. Early reckless urban planning and huge population (more than 24 million) [3] spawns enormous travel demand. According to statistics from Shentong Inc., the

\footnotetext{
* Correspondence: danielsun@sjtu.edu.cn

'State Key Laboratory of Ocean Engineering, Shanghai Jiao Tong University,

Shanghai 200240, China

${ }^{2}$ China Institute of Urban Governance, Shanghai Jiao Tong University,

Shanghai 200240, China

Full list of author information is available at the end of the article
}

operating company of Shanghai Metro, for the five continuous weekdays from February 29 to March 4, 2016, the network-wide passenger loading of Shanghai Metro were all over 10 million, attaining 10.16 million, 10.23 million, 10.33 million, 10.25 million and 10.82 million, respectively [4]. As a result, uncomfortable travel experience occurred, including huge on-train overcrowding during peak hours, which seriously affected the comfortableness of passengers, and even influenced their working efficiency and quality of lives.

For the overcrowding issue in metro system, an obvious problem is that the capability isn't always being fully utilized, i.e., some sections are heavily crowded while at the same time others may be underused. Taking AM peak as an example, traffic demand is enormous from overall perspective, however, it can't be ignored that some sections are not crowded at all. Metro system administrator can mitigate the overcrowding by increasing 
service frequency, which may bring additional issues, as 1) more trains are required which brings huge monetary cost; 2) the capacity may meet the peak demand but also induce the wasting of resources for segments where the demand is not large enough. Another feature of AM peak flow in metro system is directional non-uniformity. Lines connecting suburban and central areas, such as Line 5, and those across the city center, such as Line 2, all possess directional non-uniformity. Therefore, solely improving dispatching frequency is not effective in solving the traffic overcrowding problem.

Commuter trips have tidal feature in nature, spatial and temporal inhomogeneity and directional non-uniformity, which is prone to induce vehicle congestion/passenger overcrowding or resource wasting. To avoid these, transportation administrators should adjust the operating strategies to meet different demand. Similar situations have been appeared in the regular ground transit operation. Previous studies have proposed short turning schedule along with full-length operation policy $[5,6]$, which were also used in railway system [7, 8]. Short turning, also called short turn, relatively with full-length policy, means selecting two stations within the entire line as the new origin and destination to improve the transport capacity for the section in between. Short turning is a convenient tactical strategy when high demand sections need to be served while others only have relative low demand, with respect to simply increasing the dispatching frequency. As passenger demand can be met by various operation patterns, such as short turning and full-length policy, consequently schedule coordination between these patterns is indispensable.

Short turning is an operating pattern not only for regular public transit and railway system, but also for urban metros, which is particular efficient for lines connecting suburb residential areas with city center or connecting two main transportation hubs. The strategy has been used within other situations, such as zoning, restricted, semi-restricted service, and express service [9, 10]. To improve the frequency among specific zones of the metro line, short turning may be performed to reduce average waiting time within these sections [5]. Studies related to the short turning operation pattern within urban metro system have to coordinate with the traditional operating patterns with an objective of minimizing both the passengers' waiting time [11] and the fare costs, as well as the costs of operators [12]. Other research on short turning concerned the timetabling level [13]. Taking these into consideration, this study mainly focused on the short turning zone selection in order to relieve passenger overcrowding.

With reference to Shanghai Metro system, Line 2 is the second longest line, with $64 \mathrm{~km}$ in length (right behind Line 11, $82.3 \mathrm{~km}$ ), connecting Hongqiao International Airport and Pudong International Airport. The line passes through several important business centers, including East Nanjing Rd., People's Square, Lujiazui etc. , with rather huge traffic demand. Existing operational patterns of Line 2 already have short and long turning dispatches, as presented in Fig. 1. However, due to the setup of turn-around facilities and other external reasons, the short turning strategy can only be scheduled within certain stations. For example, trains with 8carriages can only run from East Xujing to Guanglan Rd. and the 4-carriage ones from Guanglan Rd. to Pudong International Airport because of the limitation of track facilities. This study releases turn back facilities constraints and takes passengers' utility into consideration, exploring an effective short turning operation pattern in relieving passenger overcrowding during peak hours. An optimization model is proposed to determine short turning parameters, during which the loading status was introduced to reflect the overcrowding condition.

The remainder of this paper is organized as follows. Section 2 presents literature review with research gap and proposes research methods of this paper. Mathematical programming model is formulated in Section 3, followed by a case study of Shanghai Metro Line 2 in Section 4. Finally, conclusions for future work are summarized in Section 5.

\section{Literature review}

Imbalance between urban transport supply and demand raises many problems. Jara-Díaz et al. [14] analyzed the demand between each station pair within a single bus transit line by comparing with models under different demand aggregation levels (i.e. aggregate and disaggregate demand) for obtaining optimal frequency and vehicle size. Temporal differences in passenger volumes can be addressed by providing a higher service frequency during peak hours, while the spatial demand unbalances justify the implementation of fleet assignment strategies, by increasing the service frequency on the high demanded route sections to adjust the service demand with the capacity supply $[15,16]$. To deal with this, Tirachini [17] developed a disaggregated short turning strategy with information at a station level. Short turning strategy in bus corridor often uses demand from station to station within a single line and certain period setting. The main objective is to increase the service frequency on sections with high demand, thus to deal with spatial concentration of overcrowding considering the costs of both transit agency and passengers. Frequencies (inside and outside the short cycle), capacity of vehicles and the location of short turn limited stations were determined through short turning model. To relieving overcrowding, Cortés et al. [18] developed a model that combines short turning and deadheading in an integrated strategy for a single transit line. Ulusoy and Chien [19] attempted to 


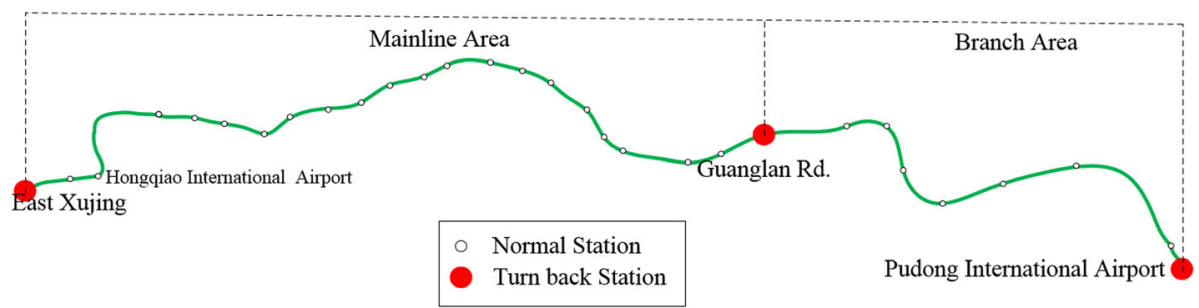

Fig. 1 Existing operating pattern of Shanghai Metro Line 2

optimize bus service patterns (i.e. all-stop, short-turn, and express) and frequencies, thus to minimize total cost, considering transfer demand elasticity, similar to Ulusoy et al. [20], Zhao and Zeng [21]. These studies are mainly applications of regular transit system without considering facility constraints.

When coming to railway system, short turning has not been widely used because railway system mainly concerns about timetable [7, 8, 22] and rolling stock [23]. Louwerse and Huisman [7] adjusted a railway timetable in case of large disruptions and presented integer programing formulations based on event-activity networks for the situations of a partial and a complete blockade. Timetable determination was also studied by means of building and solving a nonlinear integer programming model that fitted the arrival and departure time to a dynamic demand $[8,22]$. Lobo and Couto [24] investigated the relationship between the operational performance of metro systems and their socioeconomic contexts, but unfortunately no effective improvement measures were proposed.

Nagorsky et al. [25] described the development and evaluation of potential interventions to improve the rapid transit network's ability and provide service to customers in addressing subway overcrowding. Canca et al. [11] proposed a short-turning policy in rail transportation to handle the passenger overloads within train service disruptions by increasing the frequency among certain stations and equilibrating the train occupancy level. Turn-back points and service offsets were determined with the objective to minimize passengers' waiting time while ensuring a certain level of quality of service. Sun et al. [12] proposed a total cost minimization model for redesigning the short turn operation by mainly relaxing the assumption that the fulllength route must be operated with subjecting to the restriction of turn-back stations. Then, to meet the increased passenger demand and overcrowding around urban and suburban areas, an integrated planning model was proposed to adequate the offered capacity and system frequencies by Cadarso and Marín [26]. What these studies might neglect is that public transit is a human-oriented transport system, relieving overcrowding for a better travel experience is as important as reducing travel time. Parbo et al. [27] reviewed passenger perspectives in railway timetabling and emphasized the importance of passenger-oriented railway timetabling to save the passengers waiting time.

Consequently, summaries are made through the literatures as follows.

- Short turning operating policy is widely used in regular transit. However, as the ground transportation is not limited by the tracks, applications of different operating policies are relatively free.

- Timetables are the main focus in the railway system. Some complex mathematical programming models are used to obtain a better schedule.

- Urban metro systems have combined operating policies of traditional ground transit and railway systems, but unfortunately, haven't given the priority to passengers.

This study mainly focuses on relieving peak hour overcrowding of urban subway based on previous studies by relaxing the restriction of turn-back stations. A mathematical model was proposed to determine the short turning parameters, during which a load factor was introduced as a measurement of overcrowding condition. Finally, empirical data obtained from Shanghai Metro were used to demonstrate the capability of the proposed method.

\section{Mathematical model}

\subsection{Problem statement}

Metro system generally includes lines of two directions, namely up and down directions. Each train of certain line follows a back and forth route from origin to destination then turn back, and different lines are actually operated separately to a certain extent, which can be analyzed individually. Passenger demand is generally different for the stations along the line. As demonstrated in Fig. 2, passenger demand between Stations 6 and 18 (the shadow section) is significantly larger than the other stations (with the correspondence of station name and number listed in Appendix 1) during AM peak hour (07:30-08:30) and the demands of up and down directions in each station are largely different. As a result, overcrowding occurring among these stations will affect passengers' travel experience. In contrast, no large 


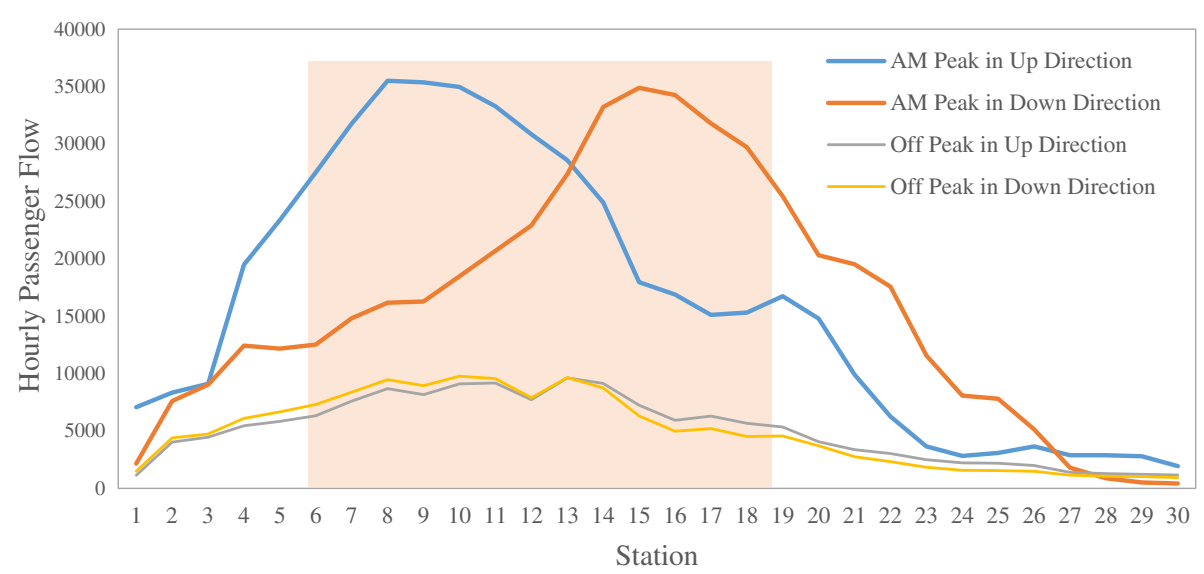

Fig. 2 Hourly passenger flow of Shanghai Metro Line 2 during AM and PM peaks (AM peak 7:30-8:30, Off Peak 14:30-15:30, Sept. 16, 2014)

difference on traffic demand and directional non-uniformity for different stations was found during PM off peak hour (14:30-15:30). Therefore, these overcrowding sections are the investigating focus of this study, in order to provide rational and efficient short turning operating policies. As short turning pattern need to be coordinated with present operation strategies, the question turns to determine the specific short turning zone and the coordinated schedule.

A regular symmetric full-length service and a short turning service between Stations 2 and $n-2$ are illustrated in Fig. 3. The vertical axis represents travel time from origin to destination and the horizontal axis represents travel distance. Overlapping area of full length and short turning service have larger transport capacity than those outside of the short turning zone. As the cumulative demand in short turning zone is larger, the short turning strategy could relieve overcrowding within the zone. With an objective to reduce overcrowding in a high traffic demand area, the following steps were conducted. First, selecting short turning zone with comparable higher traffic demand, and which is congested in peak hours. Then, defining the operating timetable, such as offset of inserting new ones to existing ones.

Although applying short turning pattern may need additional trains and larger frequency, which is generally considered as part of public competencies, the cost constraint was relaxed from passengers' welfare perspective. For simplicity, other assumptions were made as follows.

a) For both full length and short turning services, all trains have to stop at every station in between and operate at the same speed with fixed headway.

b) No turn around constraints exist along a line since turn around facilities can be built without many particular difficulties, although only a subset of stations containing short-turning facilities may be established. c) All passengers waiting on the platforms take the recent arrival train and are able to aboard.

d) Passengers arrive uniformly during the peak period regardless of stochastic demand.

\subsection{Model formulation}

Without loss of generality, a metro line can be modeled as a directed graph, $G=(V, L)$, in which $V$ denotes station (vertex) set, $L$ denotes link set. Station is denoted as $i \in V=\{1,2, \ldots, n\}$, and link, between any two consecutive stations, is denoted as $l \in L=\{1,2, \ldots, n-1\}$. Route denotes a train runs back and forth, indicated by $r \in R$. Two routes are presented in Fig. 3, one (Route 1) covers Station 1 to Station n, and the other (Route 2) covers Station 2 to Station n-2 overlapping with Route 1. Passenger demand between different stations is denoted by OD (Origin-Destination) pairs, an $n$ by $n$ matrix.

\subsubsection{Parameters}

$M$-a sufficiently large constant (chosen as 9999 during the numerical experiment);

$h$-full length headway;

$h_{\text {min }}$-the minimum headway;

$h$-the offset between short turning and full-length services;

$q_{i j}$-hourly demands from Station $i$ to Station $j$;

$u$-upstream direction;

$d$-downstream direction;

$Q_{k}^{u}$-hourly demand of Station $k$ in the upstream direction (u-up direction);

$Q_{k}^{d}$-hourly demand of Station $k$ in the downstream direction ( $d$-down direction);

Cap-capacity of each train (310 passengers per carriage unit, 6 passengers $/ \mathrm{m}^{2}$ );

$\alpha_{i j r^{-}}$binary variable, whether trips from Station $i$ to Station $j$ are covered by Route $r, 1$-yes, 0 -no; 


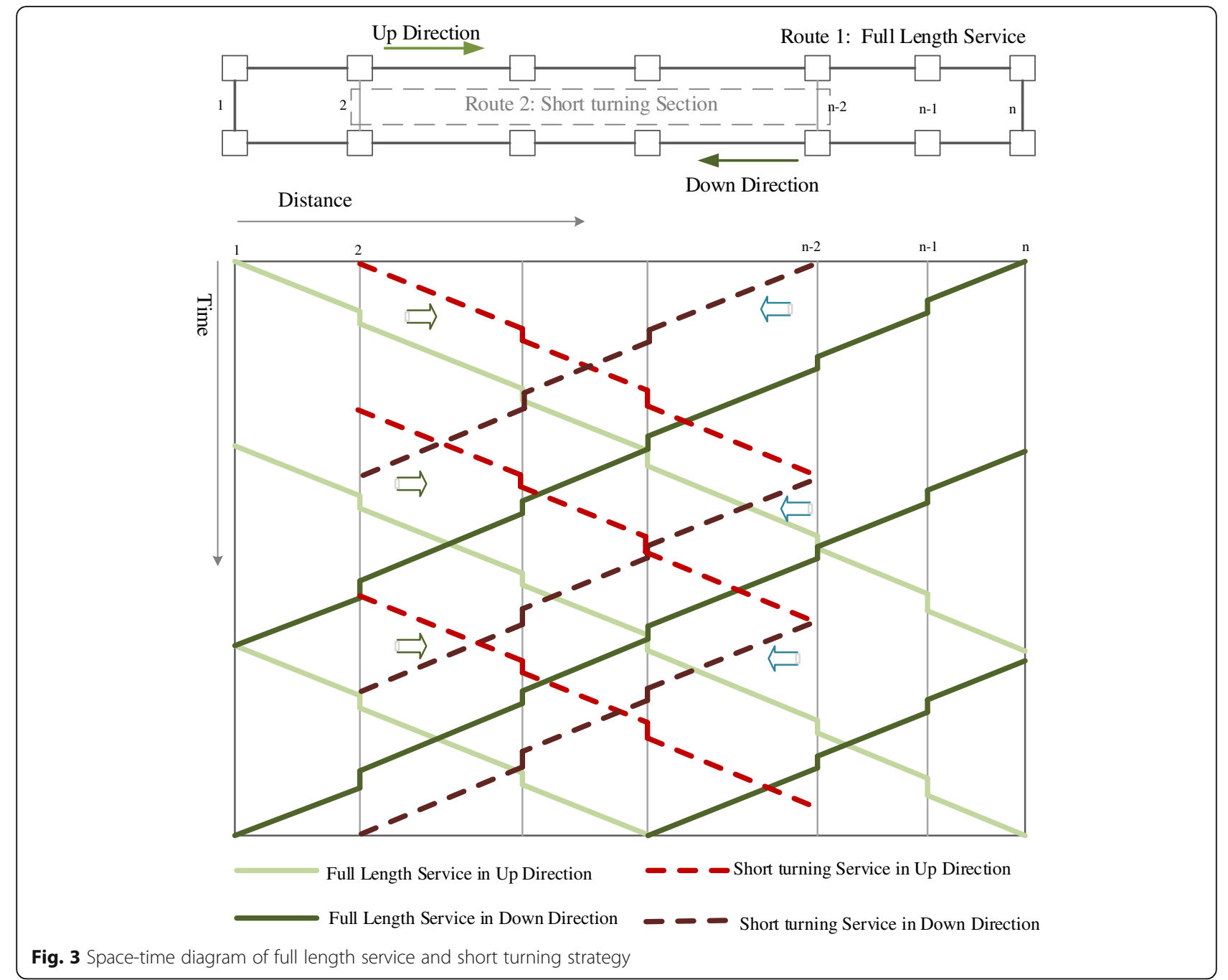

$\beta_{i j}$ - binary variable, whether passengers can reach destination $j$ directly from Station $i$, 1-yes, 0 -no;

$\gamma_{y z}$, binary variable, whether the zone from Station $y$ to Station $z$ is selected for short turning services, 1yes, 0-no;

\section{$\eta$-load factor;}

$\delta_{r k}$-binary variable, whether Route $r$ covers Station $k$, 1 -yes, 0 -no;

$\pi_{r k^{\prime}}$-binary variable, whether turn back Station $k^{\prime}$ is part of Route $r$, 1-yes, 0-no;

$\tau_{k^{\prime}}$-headway of Station $k^{\prime}$ for turning back;

$S_{r}$-binary variable, whether Route $r$ is selected, 1 -yes, 0 -no;

$h$-the offset between short-turning and full-length services, continuous.

$f_{r}$-frequency of Route $r$, continuous;

$\theta_{i j}$-whether OD pair $i$ to $j$ can be served directly without interchange.

\subsubsection{Decision variables}

Short turning zone: $r_{y z}, y, z \in V^{\prime} \in V$, route is denoted as $r \in R$;

Frequency of route $\mathrm{r}: f_{r}$.

\subsection{Constraints}

3.3.0.1 Train capacity constraint Different stationbased passenger demands exist in upstream and downstream direction because of directional non-equilibrium of passenger flow during AM peak period. Demand of up and down directions can be represented as follows, respectively:

$$
Q_{k}^{u}=\sum_{i, j \in V: i \leq k<j} q_{i j}, \forall k \in V
$$




$$
Q_{k}^{d}=\sum_{i, j \in V: j \leq k<i} q_{i j}, \forall k \in V
$$

where, $Q_{k}^{u}$ and $Q_{k}^{d}$ denote the cumulatively demand of Station $k$ in up and down direction, respectively.

If multiple operating patterns exist, such as a fulllength service together with a short-turning service, stations are actually served with different train capacity. The capacity constraints of outside and inside short turning zone are defined as follows.

$V^{\prime} \in V$ - a subset of stations containing short-turning facilities.

$h$ - the offset between short-turning and full-length services.

$\gamma_{y z}\left(y, z \in V^{\prime}: y<z\right)$, binary variable, 1 if the zone is selected for short turning services.

Then, the constraint is expressed by Eq. (3) as below:

$$
\sum_{y, z \in V^{\prime}: y<z} \gamma_{y z}=1
$$

As stations are served by different routes (full-length route and short turning route), capacity constraints of different zones are expressed as follows:

1) Full-length service outside the short-turning zone:

$$
\begin{aligned}
& Q_{k}^{u(d)} \leq \eta \operatorname{Cap} \sum_{r} f_{r} \delta_{r k}+M\left(1-\gamma_{y z}\right), y, z \in V^{\prime}: y \\
& \quad<z ; k<y \text { or } k>z
\end{aligned}
$$

2) Full-length service inside the short-turning zone:

$$
\begin{aligned}
& Q_{k}^{u(d)} \frac{h^{\prime}}{h} \leq \eta \operatorname{Cap} \sum_{r} f_{r} \delta_{r k}+M\left(1-\gamma_{y z}\right), y, z \in V^{\prime} \\
& \quad: y<z ; y \leq k \leq z
\end{aligned}
$$

3) Short-turning service:

$$
\begin{aligned}
& Q_{k}^{u(d)}\left(1-\frac{h^{\prime}}{h}\right) \leq \eta \operatorname{Cap} \sum_{r} f_{r} \delta_{r k} \\
& +M\left(1-\gamma_{y z}\right), y, z \in V^{\prime} \\
& \quad: y<z ; y \leq k \leq z
\end{aligned}
$$

where, $Q_{k}^{u(d)}, Q_{k}^{u(d)} \frac{h^{\prime}}{h}, Q_{k}^{u(d)}\left(1-\frac{h^{\prime}}{h}\right)$ denote accumulative passengers for Station $k$ in both up and down directions; Cap indicates the capacity of a train (number of passengers). $f_{r}$ denotes frequency of Route $r$, and $\delta_{r k}$ is a binary variable, indicating whether Station $k$ is covered by Route $r$. Load factor $\eta_{k}$ indicates utilization of the capacity at Station $k$. As a result, $\eta_{k} * C a p * \sum f_{r} \delta_{r k}$ indicates the practical capacity of Route $r$ during the peak hours at Station $k$.

To avoid heavy overcrowded and prevent resources wasting simultaneously, the loading factor $\eta_{k}$ of each train must be within a certain rational range, which is general low at the initial departure station and increases gradually, and then decreases. Therefore, load factor of a train indicates the maximum short-term load factor during oneway operating period. Capacity of each train is calculated based on full loaded with criterion of 6 passengers $/ \mathrm{m}^{2}$. To avoid overcrowded, the maximum load factor $\eta_{k}$ is limited to no more than 1.2. Meanwhile, to avoid resources wasting, short turning zone has to be selected from stations with an original load factor $\eta_{k} \geq 0.8$.

3.3.0.2 Line capacity constraint Each metro line within the subway system generally has a theoretically minimal headway $h_{\text {min }}$ from technical perspective (1.5 min for subway system using A-type vehicle). As presented in Eq. (7), the cumulatively frequency $\sum_{r} f_{r} \delta_{r k}$ for any station $k$ can't exceed theoretically maximum frequency $\frac{1}{h_{\min }}$.

$$
h_{\text {min }}<\frac{1}{\sum_{r} f_{r} \delta_{r k}}, \forall k \in V
$$

3.3.0.3 Turn around capacity constraint The minimum time for turning around is possibly ranging from 1 to 5 min (Canca et al., 2014b). As presented in Eq. (8), the combined frequency of any route should not exceed the maximal allowable turn-back frequency.

$$
\tau_{k^{\prime}} \leq \frac{1}{\sum_{r} f_{r} \pi_{r k^{\prime}}}
$$

\subsubsection{Objective function}

As the overcrowding status of train can be controlled by a predefined load factor $\eta$, travel time is used for evaluating travel experience depending on the departure frequency, short-turning zone selection etc. As all trains are supposed to run at the same speed, and the on-train time is identical, the only difference lies in the platform waiting time. Therefore, the passengers' waiting time was chosen as the objective of the model.

Other parameters, $\alpha_{i j r^{-}}$indicates whether trips from Station $i$ to Station $j$ are covered by Route $r$, binary variable. $S_{r}$-whether Route $r$ is selected, binary variable. $\delta_{r k^{-}}$ whether Route $r$ covers station $k$, binary variable. $\beta_{i j}$ 
$=\max _{r}\left\{\alpha_{i j r} S_{r}\right\}, \forall i, j \in V$-whether passengers can reach destination $j$ directly from Station $i$, binary variable.

If $\alpha_{i j r}=1$, passengers from Station $i$ can travel to destination $j$ directly without transferring. If $\beta_{i j}=1$, the average waiting time is calculated as below:

$$
t_{i j}=\frac{1}{2 \sum_{r} \alpha_{i j r} f_{r}}, \forall i, j \in V
$$

If $\alpha_{i j r} \cdot S_{r}=0$, based on the assumption that passengers on the platform are all able to aboard, these passengers at most have one transferring and consequently, the average waiting time is calculated as:

$$
t_{i j}=\frac{1}{2}\left(\frac{1}{\sum_{r} \delta_{r i} f_{r}}+\frac{1}{\sum_{r} \delta_{r j} f_{r}}\right), i, j \in V
$$

As a result, the objective function of model is formulated as follows:

\subsubsection{Objective function}

$\min \sum_{i} \sum_{j} q_{i j}\left(\beta_{i j} \cdot \frac{1}{2 \sum_{r} \alpha_{i j r} f_{r}}+\left(1-\beta_{i j}\right) \cdot \frac{1}{2}\left(\frac{1}{\sum_{r} \delta_{r i} f_{r}}+\frac{1}{\sum_{r} \delta_{r j} f_{r}}\right)\right)$

subject to:

Train capacity constraints: (3), (4), (5), (6).

Load factor: $0.8 \leq \max \left\{\eta_{k}, k \in V^{\prime}\right\} \leq 1.2$.

Line capacity constraint: (7)

Turn-back capacity constraint: (8)

The objective is to minimize passengers' average waiting time under necessary constraints. The train capacity constraint ensures that the supply could meet passenger demand at certain level of service. Line capacity constraint guarantees the operating frequency does not exceed the permissible value, while the turn back constraint makes trains turn around without exceeding the turn-back capacity. Load factor was highlighted here for ensuring the degree of occupancy.

\section{Numerical experiment}

\subsection{Data sources}

This section applies the proposed model by using Shanghai Metro Line 2 as an empirical case study, mainly for the largest passenger demand and the second longest line length. Line 2 is an east-west direction line of the Metro network (see Fig. 4, the light green line), running from East Xujing station in the west to Pudong International Airport station in the east, passing Hongqiao International Airport, East Nanjing Rd., People's Square, and the Lujiazui financial district in Pudong etc. with the highest average passenger occupancy rate. It is also the second longest line with a full length of $64 \mathrm{~km}$, right after the new opened Line 11 (with an overall length of $82.3 \mathrm{~km}$ ).

The eastern portion of the line, from Guanglan Rd. to Pudong International Airport, operates independently from the main line due to the infrastructure constraint, where only the 4-carriage trains were run. The western portion, also the main route, runs 8-carriage trains from East Xujing to Guanglan Rd., as shown in Fig. 1. Consequently, the two operating routes for Line 2 are the one from East Xujing Station to Guanglan Rd. Station (Stations 1 to 21) using the AC02a series trains (designed and manufactured by the German company - ADTranz \& $\mathrm{Si}$, with an operational speed limit $80 \mathrm{~km}$, VVVF AC drive and 8-carriages), and the other from Guanglan Rd. to Pudong International Airport (Stations 22 to 30) using the trains with four carriages.

Due to the high passenger demand along Line 2 during the AM peak, the train is overcrowded from Stations 6 to 18 (within the western portion of the line), as presented in Fig. 2. It was found that passenger demand is not extremely large along the eastern portion, and short turning zone is not necessary to cover this segment. Therefore, the main purpose of this case study is to determine a short turning zone with higher passenger demand both in up and down directions, as well as the dispatching frequency of the short turning trains. The objective will be achieved throughout the parameters found out using the model, i.e. Eq. (11).

Passenger OD data, for AM peak 7:30-8:30, Sep. 16, 2014 were obtained from Shentong Inc., from which the number of passengers taken Line 2 were estimated. As the train operated in the west portion of Line 2 is composed by 8 AC02a-type carriages and each accommodates 310 passengers $\left(6\right.$ passengers $\left./ \mathrm{m}^{2}\right)$ in maximal. Accordingly, the total capacity of a train in the west portion is calculated as $310 * 8=2480$ passengers. The present dispatching headway is $6 \mathrm{~min}$, and the frequency is 10 per hour. Therefore, the total capacity of the main line is $2480^{*} 10=24,800$ passengers per hour. If the accumulative passengers are close to or exceed this value, short turning pattern with an original departure headway as 6 min should be considered.

\subsection{Results analysis}

The situation was formulated into a mathematical problem based on Eq. (11)., which was solved numerically by using integer programming method. Results, including the departure and terminal stations number and the dispatching frequency of original and new routes, are presented in Table 1 . The name for each station can be obtained from Appendix.

Two original routes, one from East Xujing to Guanglan $\mathrm{Rd}$. and the other from Guanglan Rd. to Pudong International Airport, were retained in the new operation 




Fig. 4 Shanghai Metro system (in Sept. 2014)

pattern with adjustments of departure headway. A new route, namely short turning route, from Beixinjing to Longyang $\mathrm{Rd}$., is proposed to increase transport capacity within the high demand zone. Dispatching frequency of the original routes is set as 10 trains per hour consistently. It is obvious that the departure frequency between Stations 5 and 19 is $8+6=14$ trains per hour, higher than before while the others were kept unchanged or reduced. Accordingly, short turning pattern is reasonable to mitigate overcrowding, different from simply improving departure frequency. By this, overcrowding of the higher traffic demand zone is relieved on purpose without too much capacity wasting.

Figure 5 presents the details about capacity, demand and load factor for the schedule. The blue bar graph represents passenger demand in both up and down directions, and green circle and triangle line denotes the supply capacity. It can be figured out that the new supply capacity could almost satisfy the demand. Yellow circle-dotted line represents load factor of original route, red triangle-dotted line denotes the new routes' load factor. Original load factors attain as high as 1.43 , and nine stations in up direction, and seven stations in down direction are with the load factor larger than 1, indicating heavily crowded. After applying the proposed operation plan, transport capacity is enhanced significantly among the higher demand zone and the load factor is reasonably decreased. Only a few stations are still with load factor slightly exceeding 1 . Therefore, the overcrowding situation has been relieved to a large extent through incorporating the short turning pattern.

Passenger OD data used in this study was from 2014, consequently the optimization presented is mainly based on the operation strategy at that time. In fact, Shanghai Metro Line 2 has adopted short turning pattern between Songhong Rd. and Guanglan Rd. since 2016 for relieving the huge demand, as presented in Fig. 6. The red dash line is the short turning route proposed while the green dash line represents the existing operating routes. Similarities of these two short turning routes can be summarized as follows. Both are a subset of long turning routes, and containing high passenger demand stations, which further proves the validity of the results of this study. The difference of the two short turning routes

Table 1 New short turning route and original route

\begin{tabular}{lllll}
\hline Type & Departure Station Number & Terminal Station Number & Freq. (per hour) & Route Status \\
\hline Original & 1 & 22 & 10 & Full-length (western) \\
& 22 & 30 & 10 & Full-length (eastern) \\
New & 1 & 22 & 8 & Full-length (western) \\
& 5 & 19 & 6 & New \\
& 221 & 30 & 10 & Full-length (eastern) \\
\hline
\end{tabular}




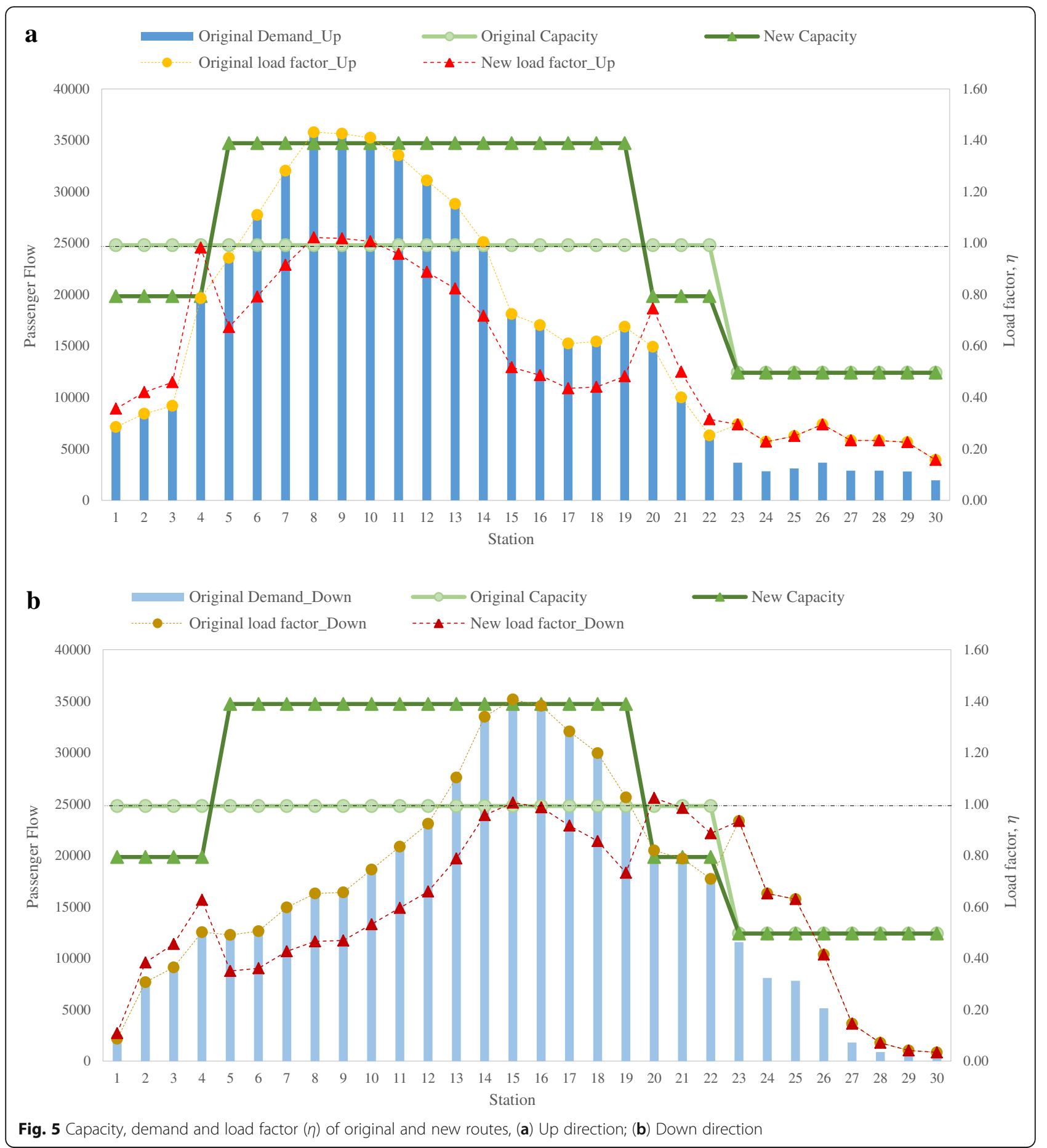

may mainly lie in the practical turning back constraints and other field operational constraints.

\section{Conclusions}

As urban commuter trips have tidal feature in nature, particularly the demand on different stations and zones is differed largely, which requires a dynamic dispatching and operational pattern on each lines of the metro system.
Although simply improving departure frequency for all routes could also relieve overcrowding, it generally brings a higher cost and results in a capacity waste within certain under-saturated stations, where the load factor becomes extreme low. Short turning pattern is effective in both relieving overcrowding and avoiding capacity wasting. This study mainly focused on relieving overcrowding of urban metro system during peak hours by introducing short 


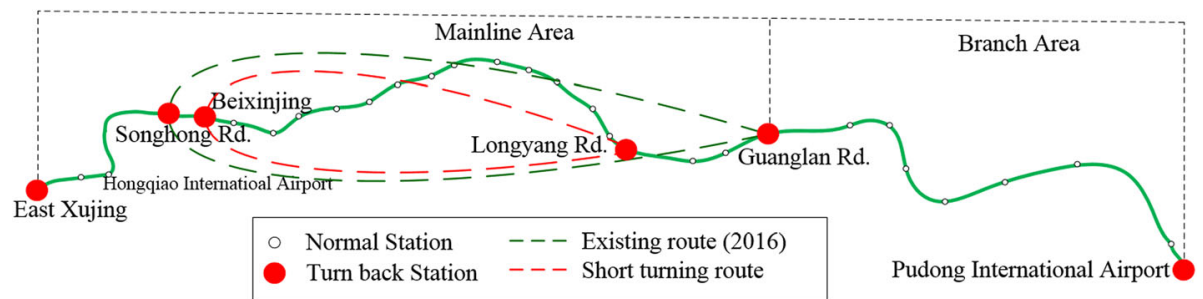

Fig. 6 Existing route (2016) and short turning route proposed

turning pattern. A mathematical model was proposed in determining short turning zone with an objective of minimizing the average waiting time of passengers. Train capacity, line capacity, and turn around capacity constraints, as well as load factor were considered. Turn back facility constraints was relaxed to select short turning zone more flexibly. Line 2, the busiest line in Shanghai Metro system, was selected as a case study for relieving overcrowding during AM peak period. A short turning route was determined which could significantly relieve overcrowded condition. The practical case study further demonstrated the effectiveness of the proposed short turning determination model. Conclusions can be summarized from the study as follows:

- Short turning pattern is an effective method for relieving overcrowding without wasting resources.

- Load factor is an influential and essential factor in determining short turning zone.

- Case study of Shanghai Metro Line 2 indicates that a short turning route from Beixinjing to Longyang $\mathrm{Rd}$. with a frequency of six trains per hour could significantly ease congestion without causing much wasting comparing with simply improving departure frequency.

Although the results are promising, additional studies may be conducted to improve model performance from following aspects. First, coordination of short turning pattern and existing operating patterns was not explored in this study. Consequently, results of the case study may be just a feasible solution rather than the global optimal solution for constraints of the existing operation plan and track infrastructure. Next, improving dispatching frequency and opening new short turning need monetary cost which is a practical and critical issue. The objective function of the proposed mathematical model unfortunately doesn't provide the quantitative monetary analysis. A CBA (Cost Benefit Analysis) considering this solution compared with the improving departure frequency could also be a further research step. Finally, with the metro capacity increases, relieving overcrowding, or more flexible working hours, some passengers may change their trip time or mode, or at least both travel time and passenger demand are stochastic in reality. To this end, the proposed model could be extended as a Markovian Process to handle these potential issues.

\section{Appendix}

Table 2 The correspondence of station number \& name of Metro Line 2, Shanghai

\begin{tabular}{llll}
\hline No. & Station name & No. & Station name \\
\hline 1 & East Xujing & 16 & Century Avenue \\
2 & $\begin{array}{l}\text { Hongqiao Railway } \\
\text { Station }\end{array}$ & 17 & Shanghai Science Technology \\
& & Museum \\
3 & Hongqiao Airport & 18 & Century Park \\
& Terminal 2 & & \\
4 & Songhong Rd. & 19 & Longyang Rd. \\
5 & Beixinjing & 20 & Zhangjiang Hi-Tech Park \\
6 & Weining Rd. & 21 & Jinke Rd. \\
7 & Loushanguan Rd. & 22 & Guanglan Rd. \\
8 & Zhongshan Park & 23 & Tangzhen \\
9 & Jiangsu Rd. & 24 & Middle Chuangxin Rd. \\
10 & Jing'an Temple & 25 & East Huaxia Rd. \\
11 & West Nanjing Rd. & 26 & Chuansha \\
12 & People's Square & 27 & Lingkong Rd. \\
13 & East Nanjing Rd. & 28 & Yuandong Avenue \\
14 & Lujiazui & 29 & Haitiansan Rd. \\
15 & Dongchang Rd. & 30 & Pudong International Airport \\
\hline
\end{tabular}

Note: the italicize boldface names: East Xujing and Pudong International Airport represent the start and end stations of the metro line 2 in Shanghai

\section{Acknowledgements}

The authors would like to express their appreciation to Drs. Zhong-Ren Peng and Yi Zhu from School of Naval Architecture Ocean and Civil Engineering, Shanghai Jiao Tong University for their valuable suggestions and assistance in this study. The research was supported in part by the Shanghai Municipal Natural Science Foundation [17ZR1445500], China, the State Key Laboratory of Rail Traffic Control and Safety [RCS2018K010], Beijing Jiaotong University, and the Humanities and Social Science Research Project [15YJCZH148], Ministry of Education, China. Any opinions, findings and conclusions or recommendations expressed in this paper are those of the authors and do not necessarily reflect the views of the sponsors.

\section{Authors' contributions}

XD proposed the initial model, performed the numerical experiment as well as result analysis, and drafted the manuscript. SG participated in the model 
implementation and calibration, as well as the design and data preparation of numerical experiment. $\sqcup$ participated in the design of this study and the outcome analysis. DJS conceived of the entire framework of the study and participated in its design and coordination. All authors read and approved the final manuscript.

\section{Competing interests}

The authors declare that they have no competing interests.

\section{Publisher's Note}

Springer Nature remains neutral with regard to jurisdictional claims in published maps and institutional affiliations.

\section{Author details}

${ }^{1}$ State Key Laboratory of Ocean Engineering, Shanghai Jiao Tong University, Shanghai 200240, China. ${ }^{2}$ China Institute of Urban Governance, Shanghai Jiao Tong University, Shanghai 200240, China. ${ }^{3}$ Shanghai Urban Construction Design and Research General Institute, Shanghai 200125, China. ${ }^{4}$ State Key Laboratory of Rail Traffic Control and Safety, Beijing Jiaotong University, Beijing 100044, China.

Received: 2 October 2017 Accepted: 5 April 2018

Published online: 15 June 2018

\section{References}

1. Sun DJ, Guan S (2016) Measuring vulnerability of urban metro network from line operation perspective. Transp Res A: Policy Pract 94:348-359. https://doi.org/10.1016/j.tra.2016.09.024

2. Sun DJ, Zhao Y, Lu Q (2015) Vulnerability analysis of urban rail transit networks: a case study of shanghai, China. Sustainability 7(6):6919-6936. https://doi.org/10.3390/su7066919

3. Shanghai Municipal People's Government. Statistical Yearbook of Shanghai (In Chinese). http://www.shanghai.gov.cn/nw2/nw2314/nw24651/nw42131/ nw42137/u21aw1230847.html, Accessed 12 Dec 2017

4. Jin, Z. (2016). New norm: Metro passenger flow exceeds ten million on workday. Xinmin Evening News, A13 (In Chinese). http://xmwb.xinmin.cn/ html/2016-03/08/content_13_4.htm, Accessed 16 Oct 2016

5. Furth PG (1987) Short turning on transit routes. Transp Res Record J Transp Res Board 1108:42-52 Retrieved from http://trid.trb.org/view/288816

6. Chen, M., \& Niu, H. (2009). Modeling transit scheduling problem with short-turn strategy for a congested public bus line. Eighth International Conference of Chinese Logistics and Transportation Professionals, 4320-4326. https:/doi.org/10.1061/40996(330)631

7. Louwerse I, Huisman D (2014) Adjusting a railway timetable in case of partial or complete blockades. Eur J Oper Res 235(3):583-593. https://doi.org/10.1016/j.ejor.2013.12.020

8. Canca D, Barrena E, Algaba E, Zarzo A (2014a) Design and analysis of demand-adapted railway timetables. J Adv Transp 48(2):119-137. https://doi.org/10.1002/atr.1261

9. Furth PG, Day FB (1985) Transit routing and scheduling strategies for heavydemand corridors. Transp Res Record J Transp Res Board 1011:23-26 Retrieved from http://trid.trb.org/view/271167

10. Chen S, Sun DJ (2017) A multistate-based travel time schedule model for fixed transit route. Transp Letters. https://doi.org/10.1080/19427867.2016.1271546

11. Canca D, Barrena E, Laporte G, Ortega FA (2014b) A short-turning policy for the management of demand disruptions in rapid transit systems. Ann Oper Res:1-22. https://doi.org/10.1007/s10479-014-1663-X

12. Sun Y, Schonfeld PM, Lu Y, Zhou M (2016) Redesigning rail transit short-turn operations: case study of line 2 of the shanghai metro in China. Transp Res Record J Transp Res Board 2540:46-55. https://doi.org/10.3141/2540-06

13. Site PD, Filippi F (1998) Service optimization for bus corridors with short-turn strategies and variable vehicle size. Transp Res A Policy Pract 32(1):19-38. https://doi.org/10.1016/S0965-8564(97)00016-5

14. Jara-Díaz S, Tirachini A, Cortés CE (2008) Modeling public transport corridors with aggregate and disaggregate demand. J Transp Geogr 16(6):430-435. https://doi.org/10.1016/j.jtrangeo.2008.06.006

15. Tirachini, A., \& Cortés, C. E. (2007). Disaggregate modeling of preplanned short-turning strategies in transit corridors. Transportation Research Board $86^{\text {th }}$ Annual Meeting. Retrieved from http://trid.trb.org/view/802730
16. Sun DJ, Chen S, Zhang C, Shen S (2016) A bus route evaluation model based on GIS and super-efficient data envelopment analysis. Transp Plan Technol 39(4):407-423. https://doi.org/10.1080/03081060.2016.1160582

17. Tirachini A, Cortés CE, Jara-Díaz SR (2011) Optimal design and benefits of a short turning strategy for a bus corridor. Transportation 38(1):169-189. https://doi.org/10.1007/s11116-010-9287-8

18. Cortés CE, Jara-Díaz S, Tirachini A (2011) Integrating short turning and deadheading in the optimization of transit services. Transp Res A: Policy Pract 45(5):419-434. https://doi.org/10.1016/j.tra.2011.02.002

19. Ulusoy Y, Chien SIJ (2015) Optimal bus service patterns and frequencies considering transfer demand elasticity with genetic algorithm. Transp Plan Technol 38(4):409-424. https://doi.org/10.1080/03081060.2015.1026101

20. Ulusoy Y, Chien S, Wei CH (2010) Optimal all-stop, short-turn, and express transit services under heterogeneous demand. Transp Res Record J Trans Res Board 2197:8-18. https://doi.org/10.3141/2197-02

21. Zhao F, Zeng X (2008) Optimization of transit route network, vehicle headways and timetables for large-scale transit networks. Eur J Oper Res 186(42):841-855. https://doi.org/10.1016/j.ejor.2007.02.005

22. Chierici A, Cordone R, Maja R (2004) The demand-dependent optimization of regular train timetables. Electron Notes Discrete Math 17(17):99-104. https://doi.org/10.1016/j.endm.2004.03.017

23. Alfieri A, Groot R, Kroon L, Schrijver A (2006) Efficient circulation of railway rolling stock. Transp Sci 40(3):378-391. https://doi.org/10.1287/trsc.1060.0155

24. Lobo A, Couto A (2016) Technical efficiency of European metro systems: the effects of operational management and socioeconomic environment. Networks Spatial Economics 16(3):723-742. https:/doi.org/10.1007/s11067-015-9295-5

25. Nagorsky B, Casello J, Shalaby A (2015) Strategies to relieve subway crowding: case study from the Toronto context. Transportation Research Board 94th Annual Meeting. Retrieved from http://trid.trb.org/view/1337146

26. Cadarso L, Marín Á (2012) Integration of timetable planning and rolling stock in rapid transit networks. Ann Oper Res 199(1):113-135 https://doi.org/10.1007/s10479-011-0978-0

27. Parbo J, Nielsen OA, Prato CG (2016) Passenger perspectives in railway timetabling: a literature review. Transp Rev:1-27. https://doi.org/10.1080/01441647.2015.1113574

\section{Submit your manuscript to a SpringerOpen ${ }^{\circ}$ journal and benefit from:}

- Convenient online submission

- Rigorous peer review

- Open access: articles freely available online

- High visibility within the field

- Retaining the copyright to your article

Submit your next manuscript at $>$ springeropen.com 